\title{
10-year study shines a light on grey matter atrophy in MS
}

Multiple sclerosis (MS) has traditionally been classified as a white matter disease, but growing evidence indicates that grey matter atrophy in the brain is also a prominent feature of this condition. A new longitudinal imaging study employing a technique known as source-based morphometry (SBM) has revealed specific patterns of grey matter atrophy that correlate with disability progression in patients with MS.

"SBM is a data-driven technique that relies on the use of independent component analysis - a multivariate method that identifies common spatial patterns in the data," explains lead author Niels Bergsland. "We undertook the current project in an attempt to better characterize the specific spatial characteristics of grey matter atrophy in patients with MS."

Bergsland and colleagues analysed brain MRI scans taken in 152 patients with relapsing-remitting MS, at baseline and after 10 years. The participants were divided into two groups according to the presence or absence of confirmed disability progression (CDP) at the end of the study ( $n=85$ and $n=67$, respectively).

Using SBM, the researchers identified eight distinct patterns of grey matter volume covariation at baseline, and they found that the presence of CDP after 10 years was associated

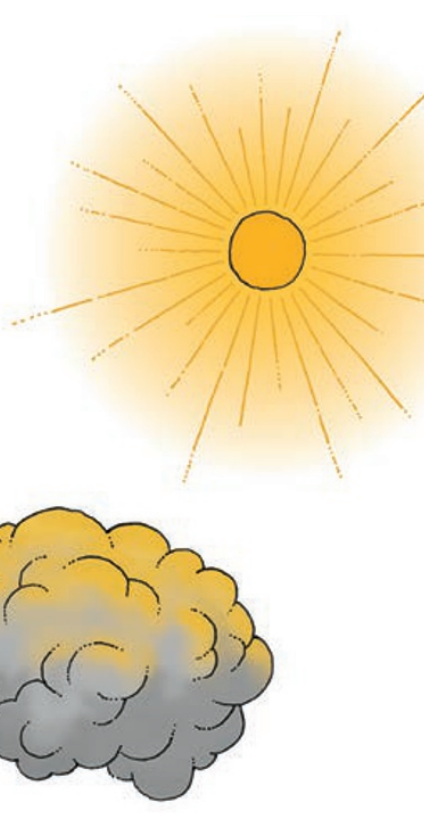

with marked atrophy in two of these patterns. One of the affected patterns included brain regions involved in motor control, whereas the other encompassed regions that are implicated in cognitive functions such as learning and memory. These findings are consistent with a previously identified link between disability and cognitive impairment in patients with MS.

"In the current paper, we only used data from a subset of patients who had imaging data at both baseline and 10-year follow-up," comments Bergsland. "However, the data set also includes annual imaging and clinical assessments, and our next step will

be to use all the available data rather than limiting ourselves to just two time points."

Heather Wood

ORIGINAL ARTICLE Bergsland, B. et al. Gray matter atrophy patterns in multiple sclerosis: a 10-year source-based morphometry study. Neuroimage Clin. 17, 444-451 (2017) 